\title{
FUNDAMENTOS PEDAGÓGICOS Y COMUNICACIONALES EN POLÍTICAS EDUCATIVAS DE ATENCIÓN DE SECTORES DE JUVENTUDES DE VULNERADOS
}

\author{
Enrique Néstor Bambozzi ${ }^{1}$
}

\section{Resumen:}

El debate en torno a la posibilidad de enunciación de pedagogías críticas desde Latinoamérica se inscribe en una línea de investigación que, en el presente, está radicada en la SECYT -UNC (Facultad de Ciencias de la Comunicación) por un equipo conformado por docentes investigadores de la Universidad Nacional de Córdoba y de la Universidad Provincial de Córdoba-Argentina. En esta etapa de la investigación, se propone logar una identificación de las perspectivas educativas y comunicacionales que configuran los procesos de escolarización del Programa de Inclusión y Terminalidad de la Educación Secundaria para Jóvenes de 14 a 17 años (PIT) en una escuela pública urbano-periférica y en el Centro Socio Educativo Penal Juvenil -Complejo Esperanza -de Córdoba. A través de un diseño cualitativo y de una investigación de tipo exploratoria y descriptiva se trabaja mediante entrevistas en profundidad a directivos, docentes y estudiantes, análisis documental y observaciones de campo indagando y sistematizando en las perspectivas educativas y comunicacionales que configuran los procesos de escolarización de educación media de jóvenes de sectores vulnerados. Nuestra pregunta se construye sobre la posibilidad de identificar diversas perspectivas educativas y comunicacionales en los procesos de escolarización acontecidos en el Programa de Inclusión y Terminalidad de la Educación Secundaria para Jóvenes de 14 a 17 años (PIT) de una escuela pública de gestión estatal y en el Centro Socio Educativo Complejo Esperanza (Penal Juvenil) de Córdoba y que estas identificaciones aportarán claves interpretativas y de intervención en torno los obstáculos y posibilidades de la ampliación o universalización de la educación secundaria a los jóvenes de sectores vulnerados como así también la toma de decisiones alternativas en la práctica de la enseñanza.

Palabras clave: adolescentes, escuela secundaria, poblaciones vulnerables

Historial del artículo:

Fecha de recibido: 11-12-2020 - Fecha de aceptado: 25-02-2021

(c) 2021 Fundación Universitaria Juan N. Corpas. FUJNC.

Artículo Open Access bajo la Licencia Creative Commons Atribución-NoComercial-Compartirlgual 4.0 Internacional. DOI: 10.26752/revistaparadigmassh.v2i2.513

${ }^{1}$ Vicerrector, Universidad Provincial de Córdoba. Profesor/Investigador Universidad Nacional de Córdoba y Universidad Católica de CórdobaArgentina. Especialista en Pedagogía. Doctor en Ciencias de la Educación. Autor para correspondencia. Correo electrónico: enriquebambozzi@ gmail.com 


\title{
PEDAGOGICAL AND COMMUNICATIONAL FOUNDATIONS IN EDUCATIONAL POLICIES FOR THE ATTENTION OF VULNERABLE YOUTH SECTORS
}

\begin{abstract}
The debate around the possibility of enunciating critical pedagogies from Latin America is part of a line of research that, at present, is based in the SECYT-UNC (Faculty of Communication Sciences) by a team made up of research professors from the National University of Córdoba and the Provincial University of Córdoba. At this stage of the research, it is proposed to achieve an identification of the educational and communicational perspectives that configure the schooling processes of the Inclusion and Termination Program of Secondary Education for Young People from 14 to 17 years old (PIT) in an urban public school- peripheral and in the Centro Socio Educativo Penal Juvenil - Complejo Esperanza - de Córdoba. Through a qualitative design and an exploratory and descriptive research, work is carried out through in-depth interviews with managers, teachers and students, documentary analysis and field observations, investigating and systematizing the educational and communicational perspectives that configure the schooling processes of secondary education of young people from vulnerable sectors.
\end{abstract}

Keywords: adolescent, secondary school, vulnerable populations 


\section{Introducción}

La investigación está radicada en la SECYT - UNC y el equipo conformado por docentes investigadores de la UNC y de la Universidad Provincial de Córdoba. En esta etapa, se propone logar una identificación de las perspectivas educativas y comunicacionales que configuran los procesos de escolarización del Programa de Inclusión y Terminalidad de la Educación Secundaria para Jóvenes de 14 a 17 años (PIT) en una escuela pública urbano-periférica y en el Centro Socio Educativo Penal Juvenil -Complejo Esperanza -de Córdoba. A través de un diseño cualitativo y de una investigación de tipo exploratoria y descriptiva se trabaja mediante entrevistas en profundidad a directivos, docentes y estudiantes, análisis documental y observaciones de campo indagando y sistematizando en las perspectivas educativas y comunicacionales que configuran los procesos de escolarización de educación media de jóvenes de sectores vulnerados. Hipotetizamos que será posible identificar diversas perspectivas educativas y comunicacionales en los procesos de escolarización acontecidos en el Programa de Inclusión y Terminalidad de la Educación Secundaria para Jóvenes de 14 a 17 años (PIT) de una escuela pública de gestión estatal y en el Centro Socio Educativo Complejo Esperanza (Penal Juvenil) de Córdoba y que estas identificaciones aportarán claves interpretativas y de intervención en torno los obstáculos y posibilidades de la ampliación o universalización de la educación secundaria a los jóvenes de sectores vulnerados como así también la toma de decisiones alternativas en la práctica de la enseñanza. En este escrito presentaremos un avance del marco teórico.

\section{Marco teórico y cuestiones metodológicas}

El problema estudiado se inscribe en la línea que los Informes Internacionales y Nacionales señalan como prioritarios en la definición de programas y proyectos para jóvenes de sectores vulnerados y el derecho a la educación media como así también las posibles traducciones en formatos pedagógicos inclusivos (Dussel (2017); Perazza (2014); Bambozzi y otros (2016 y 2017; Schapira 2016; Tiramonti (2004); Terigi (2014)). Entre algunos antecedentes , la identificación de formatos pedagógicos para la inclusión y su traducción en dispositivos de formación es un problema que afecta a la Región Latinoamericana en general y que, según informes internacionales, requiere investigaciones interdisciplinares (Informe Reimers (2004); Metas 2021 (2008); SITEAL (2013; 2015); ); Bambozzi y Vadori (2011); Coppari y Aimino (2011); Paredes y Riva (2011); Siede (2014; 2016); Bambozzi y otros (2013; 2015);UNICEF-FLACSO (2107); Ministerio de Educación de la Provincia de Córdoba (2016), entre otros. En el caso de nuestro país, estudios aislados provenientes de las Carteras Educativas tanto a nivel nacional como jurisdiccional, intentan activar -con resultados dispares -, la conformación de proyectos y programas de inclusión educativa para jóvenes de sectores vulnerados (Programas PIIE; PIT; PID, etc.).

Sin embargo, la ausencia de un marco consensuado en torno a las dimensiones constitutivas en lo teórico educativo y comunicacional necesarias para desarrollar y sostener estos proyectos, se torna en un problema reconocido tanto por docentes como por directivos y funcionarios del sistema educativo (Ministerio de Educación, 2014 y 2015; 2017). No sólo es un problema 
de operacionalización de formatos sino las nuevas perspectivas teóricas que los mismos conllevan. En este sentido, la investigación se inscribe en el campo interdisciplinario de la educación y comunicación denominado, entre otros, como comunicación educativa (J.M. Barbero), educomunicación (Mario Kaplún), Educación en Comunicación (D.P. Castillo; J.A. Huergo) que focalizan en modelos o perspectivas comunicacionales con énfasis en los procesos, en los contenidos o en los productos.

En este sentido, en el siguiente cuadro presentamos algunos avances con relación a las perspectivas comunicacionales (modelos de comunicación y dimensiones de análisis). 


\begin{tabular}{|c|c|c|c|}
\hline DIMENSIONES & $\begin{array}{l}\text { Énfasis en los } \\
\text { contenidos }\end{array}$ & $\begin{array}{l}\text { Énfasis en los } \\
\text { efectos }\end{array}$ & $\begin{array}{l}\text { Énfasis en el } \\
\text { proceso }\end{array}$ \\
\hline DIMENSIONES & Bancaria & Manipuladora & $\begin{array}{l}\text { Liberadora } \\
\text { transformadora }\end{array}$ \\
\hline PEDAGOGÍA & Exógeno & Exógeno & Endógeno \\
\hline LUGAR DEL EDUCANDO & Objeto & Objeto & Sujeto \\
\hline $\begin{array}{c}\text { CARACTERISTICAS DEL } \\
\text { ESTUDIANTE }\end{array}$ & $\begin{array}{l}\text { - Memorístico } \\
\text { - Atento } \\
\text { - Copista } \\
\text { - Subordinado }\end{array}$ & $\begin{array}{l}\text { - Pasivo } \\
\text { - Dependiente }\end{array}$ & $\begin{array}{l}\text { - Activo } \\
\text { - Pregunta } \\
\text { - Investiga }\end{array}$ \\
\hline DOCENTE & $\begin{array}{l}\text { - Función de enseñante } \\
\text { - Autoritario } \\
\text { - severo } \\
\text { - rígido }\end{array}$ & $\begin{array}{l}\text { - Instructor } \\
\text { - Es la autoridad y la } \\
\text { toma de desiciones } \\
\text { - Figura central del } \\
\text { problema }\end{array}$ & $\begin{array}{l}\text { - Social } \\
\text { - Educador que se } \\
\text { convierte a su vez en } \\
\text { educando }\end{array}$ \\
\hline OBJETIVO & $\begin{array}{l}\text { Enseñar/Aprender } \\
\text { (repetir) }\end{array}$ & Entrenar/Hacer & Pensar/Transformar \\
\hline FUNCIÓN EDUCATIVA & $\begin{array}{l}\text { Transmisión de } \\
\text { contenidos }\end{array}$ & $\begin{array}{l}\text { Moldeamiento de la } \\
\text { conducta y } \\
\text { transmisión de valores, } \\
\text { conocimientos, } \\
\text { códigos, destrezas, } \\
\text { competencias. Se basa } \\
\text { en el mecanismo } \\
\text { estímulo-respuesta }\end{array}$ & $\begin{array}{l}\text { Reflexión-Acción. Formar } \\
\text { al sujeto para que éste } \\
\text { transforme su realidad. } \\
\text { Que el sujeto: } \\
\text { - Aprenda a aprender } \\
\text { - Sea capaz de razonar } \\
\text { por si mismo } \\
\text { - Desarrolla capacidad de } \\
\text { deducción }\end{array}$ \\
\hline $\begin{array}{l}\text { TEORÍA DE LA } \\
\text { COMUNICACIÓN } \\
\text { RELACIONADA }\end{array}$ & $\begin{array}{c}\text { Modelo teórico } \\
\text { matemático de Shannon } \\
\text { y Weaver }\end{array}$ & $\begin{array}{l}\text { Modelo bidireccional de } \\
\text { Lazardsfeld y Merton }\end{array}$ & $\begin{array}{l}\text { Modelo de comunicación de la } \\
\text { corriente Latinoamericana } \\
\text { (Pasquali, Kaplun, Freire, etc.) }\end{array}$ \\
\hline
\end{tabular}




\begin{tabular}{|c|c|c|c|}
\hline $\begin{array}{c}\text { TIPO DE } \\
\text { COMUNICACIÓN }\end{array}$ & $\begin{array}{l}\text { - Transmisión de } \\
\text { información } \\
\text { (Monólogo) } \\
\text { - Relación comunicativa: } \\
\text { vertical, unidireccional } \\
\text { y autoritaria } \\
\text { - Proceso lineal: Emisor } \\
\text { activo- receptor pasivo } \\
\text { - Centrado sólo en la } \\
\text { decodificación del } \\
\text { mensaje emitido. }\end{array}$ & $\begin{array}{l}\text { - Información } \\
\text { - Comunicación como } \\
\text { herramienta de } \\
\text { persuasión. } \\
\text { - Se trata de convencer, } \\
\text { de manejar, de } \\
\text { condicionar al sujeto } \\
\text { para que adopte la } \\
\text { nueva conducta } \\
\text { propuesta. } \\
\text { - Existe un feedback } \\
\text { que le sirve al emisor } \\
\text { como instrumento de } \\
\text { verificación y control. }\end{array}$ & $\begin{array}{c}\text { Comunicación a través } \\
\text { del diálogo social. }\end{array}$ \\
\hline $\begin{array}{c}\text { ESQUEMA } \\
\text { COMUNICATIVO }\end{array}$ & $\begin{array}{l}\text { Transmisión de información } \\
\text { de un emisor }(E) \text { que envía } \\
\text { un mensaje }(M) \text { a un } \\
\text { receptor }(R) \text {. }\end{array}$ & $\begin{array}{l}\text { Sigue habiendo un } \\
\text { emisor protagonista, } \\
\text { dueño de la } \\
\text { comunicación, que } \\
\text { envía un mensaje a un } \\
\text { receptor que continúa } \\
\text { reducido a un papel } \\
\text { subordinado; pero } \\
\text { ahora aparece una } \\
\text { respuesta, } \\
\text { denominada } \\
\text { retroalimentación } \\
\text { (feedback) la cual es } \\
\text { recogida por el emisor. }\end{array}$ & $\begin{array}{l}\text { Relación comunitaria } \\
\text { humana que consiste } \\
\text { en la emisión/ } \\
\text { recepción de mensajes } \\
\text { entre interlocutores } \\
\text { (ÉMEREC) en estado } \\
\text { total de reciprocidad }\end{array}$ \\
\hline MOTIVACIÓN & $\begin{array}{l}\text { Individual: } \\
\text { premios/castigos }\end{array}$ & $\begin{array}{l}\text { Individual: } \\
\text { Estímulo / recompensa }\end{array}$ & Facilitador/Animador \\
\hline $\begin{array}{c}\text { GRADO DE } \\
\text { PARTICIPACIÓN }\end{array}$ & Mínima & Pseudoparticipación & Máxima \\
\hline $\begin{array}{l}\text { FORMACIÓN DE LA } \\
\text { CRITICIDAD }\end{array}$ & Bloqueada & Evitada & Altamente estimulada \\
\hline
\end{tabular}

Cuadro elaborado por Labandíbar, Martínez y Panero en el marco del equipo de investigación (2015-2016) como parte de su trabajo de tesis. 
Con relación a los estudios sobre las teorías pedagógicas y su articulación con el campo de la comunicación y específicamente en el orden nacional, se toman como antecedente los trabajos de Saviani (1982) y Silber (2009) en torno a las teorías críticas y no críticas de la educación cuyo criterio clasificatorio se centra en la relación educación y sociedad a partir del reconocimiento o no de la fuerza condicionante que ejercen sobre los individuos las relaciones de poder que conforman la estructura social.

Según Silber (2007) en la hoy Ilamada posmodernidad persisten y se afianzan tendencias pedagógicas que desvinculan a la escuela de cuestiones de poder y desde un pertinaz voluntarismo confían en que desde ella se habrá de cambiar la sociedad. Son las llamadas no críticas, tradicionales, neutras, "científicas" y que la autora distingue como conservadoras, tecnicistas y reformistas. Parten de una visión armónica de la sociedad y realizan un planteo voluntarista de tipo técnico-pedagógico.

En oposición, las teorías críticas cuestionan, por un lado, las de carácter idealista que postularon que a través de la escuela se conseguiría la igualación social; y por otro, las teorías sociológicas estructuralistas que afirmaron la dependencia absoluta de la educación respecto de la sociedad, definiendo la escolarización desde la lógica restringida de la dominación económica y la imposición ideológica. Su perfil actual se define en las posturas neomarxistas y posmarxistas (o poscríticas). Para nuestro estudio, utilizaremos esta perspectiva teórica como marco interpretativo referencial de los proyectos y programas de inclusión educativa.
El objetivo general propuesto es analizar las perspectivas educativas y comunicacionales que configuran los procesos de escolarización del PIT de una escuela pública de gestión estatal y en el Centro Socio Educativo Complejo Esperanza (Penal Juvenil) de Córdoba como política inclusiva de democratización del derecho a la educación de jóvenes de sectores vulnerados. Con relación a los objetivos específicos proponemos: 1) Identificar las perspectivas teóricas que sustentan el diseño, implementación y evaluación de las prácticas de escolarización en los PIT y su vinculación con lo educativo y comunicacional; 2) Reconocer y describir los distintos formatos escolares que configuran las prácticas escolares en los PIT de Escuelas Públicas de Gestión Estatal y los del Centro Socio Educativo Penal Juvenil.

La perspectiva de investigación elegida, en función de las características de nuestro objeto de estudio, es la cualitativa. Dicha metodología es apropiada, según Denzin y Lincoln (1994), cuando el investigador se propone indagar acerca de la construcción social de significados a partir de la perspectiva de los actores, sobre los condicionantes de la vida cotidiana o brindar una descripción detallada de la realidad. El alcance de la investigación es de tipo exploratoriodescriptivo. Con relación a las técnicas de recolección de datos de acuerdo con Emanuelli y Egidos [et al] (2012), las mismas se engloban dentro de los métodos y tienen un carácter operativo, se sitúan a nivel de los hechos o de las etapas prácticas que permiten la aplicación de dicho método. Son un conjunto de reglas de procedimiento y operaciones concretas utilizadas para recolectar información en el contexto de la investigación y tienen una relación directa con el objeto de estudio y 
los objetivos planteados. Operan a través de instrumentos, que son objetos concretos que facilitan la aplicación precisa de la técnica. En nuestro estudio, trabajaremos con: entrevista, observación y análisis documental. Utilizamos aquellas denominadas semi-estructuradas, que tal como definen Emanuelli y Egidos [et al] (2012), son las que se desarrollan a partir de un guión de preguntas abiertas realizadas a los entrevistados pero dejando lugar para su libre expresión. Por lo tanto, el guión no es rígido, debe servir como guía de los temas a cubrir.

Nos proponemos utilizar esta técnica como forma de encontrarnos con el otro y acercarnos a sus experiencias, sentimientos, a sus valoraciones, a su historia. Para cumplir con los objetivos de este trabajo, entrevistaremos a docentes, directivos y estudiantes. En el marco de una investigación "observar es la acción de percibir y registrar fenómenos, expresiones y manifestaciones que ocurren en un lugar y un tiempo determinado, con el propósito de construir datos para la producción de conocimiento científico" (Emanuelliy Egidos [et al], 2012, p.37). Se trata de una estrategia metodológica que, realizada en forma consciente, deliberada y sistemática, permite abordar determinados problemas de investigación o analizar perspectivas de un fenómeno social. En el caso de este estudio, la observación que aplicamos es de tipo participante, ya que tomamos contacto con el objeto de estudio desde una implicación con lo observado.

Así mismo, utilizamos observación semiestructurada, planteando una grilla que va a guiar la aplicación de la técnica, pero dejando margen a recabar datos que puedan tornarse importantes durante el trabajo de campo.
Con relación al análisis de contenidos de los documentos, se trata de una técnica que posibilita obtener información acerca de la realidad en estudio a partir de la observación y del análisis de los documentos producidos en el seno de la institución educativa. Su valor radica en que nos proporciona el punto de vista de la institución y la manera en que ésta se auto reconoce y se presenta ante la sociedad. Aplicaremos este análisis a aquella documentación que nos aportan los miembros de la comunidad educativa y constituye una fuente de información pertinente para nuestro estudio. Teniendo en cuenta que el universo o población de análisis consiste en los individuos, organizaciones, documentos y/o situaciones de las cuales se obtienen información para construir la evidencia empírica, en nuestro caso, son los PIT seleccionados.

\section{A modo de cierre}

Si bien lo expuesto da cuenta de un avance vinculado al marco referencial teórico y metodológico, varios miembros del equipo tenemos vinculación con los PIT propuestos como unidades de análisis por lo que "por fuera de la investigación en cuestión" somos portadores de sentidos construidos en el marco de nuestros recorridos por el campo empírico (instituciones, sujetos, normativas, territorios).

En este sentido, el diálogo e interpelación desdeloteórico,vaperfilandoenestemomento del estudio nuevas problematizaciones en torno a la relación entre educación y sociedad como así también la cuestión de los procesos de escolarización desde concepciones diversas y no homogéneas. 
Con relación a los aportes tanto al campo educativo como campo de conocimiento como al sistema educativo, proponemos algunos ejes de discusión desde la perspectiva pedagógica crítica.

- Educación como campo de conocimiento, campo disciplinar que - como construcción socio histórica -, tiene que seguir disputando sentidos (socialización de las producciones).

- Educación y la problematización de la habilitación de la palabra como derecho.

- Educación y la problematización de la construcción de espacios de sentido colectivos (la dimensión colectiva de la producción de sentido).
- Educación y la problematización de los procesos de escolarización como espacios de construcción de lo público.

- Problematizar la dimensión política (transformadora) de los actores escolares (justicia educativa).

- Discusión entre teorías de la educación y ¿teorías?, de la escolarización, entre otras discusiones.

Apostamos a construir nuevos sentidos que puedan traducirse en nuevas formas y formatos educativos en el marco de Políticas Públicas de Inclusión parajóvenes en contexto de vulnerabilidad.

\section{Referencias Bibliográficas}

1. Achili, Elena., (1988): "Práctica docente: una interpretación desde los saberes del maestro", en "Cuadernos de Antropología Social” N 2, FFyL, Bs.As.

2. Bambozzi, Enrique y otros. (2013) De qué hablamos cuando hablamos de Democracia Escolar. El Copista. Córdoba

3. Bonetto, María Susanay Piñero, María Teresa. (2000). El conocimiento de lo político. Advocatus. Córdoba.

4. Denzin y Lincoln (1994) "Introduction:Entering the field of qualitative research". En: Denzin, N. K., Lincoln Y.S. (eds) "Handbook of Qualitative Research" Cap. 1 Sage Publications, California. Pág. 1-17. Traducción de Mario E. Perrone

5. Dubet, François (2005) La escuela de las oportunidades ¿Qué es una escuela justa? Gedisa.

6. Dussel, Inés La Igualdad y la Inclusión en la Educación Argentina. X Jornadas de Investigación en Educación. UNC., FFyH. 5 y 6 de octubre de 2017.

7. Emanuelli, P., Egidos, D., Von Sprecher, R., Ortúzar, I., García Lucero, D., Dorado, C. y Ulla, C. (2012) "Herramientas de metodología para investigar en comunicación, conceptos, reflexiones y ejercicios prácticos". Tomo Nº 2.1 Córdoba: Editorial Copy Rápido 
7. Emanuelli, P., Egidos, D., Von Sprecher, R., Ortúzar, I., García Lucero, D., Dorado, C. y Ulla, C. (2012) "Herramientas de metodología para investigar en comunicación, conceptos, reflexiones y ejercicios prácticos". Tomo № 2.1 Córdoba: Editorial Copy Rápido

8. Jodelet, Denise (2003). Vigencia de las representaciones sociales y su incidencia en las prácticas profesionales. Entrevista realizada por María Raquel Popovich el 18 de octubre de 2003. Buenos Aires. Mimeo.

9. Labandíbar, L; Martínez, M y Panero, L (2017). Qué prácticas democráticas se construyen en la escuela. Una mirada desde el campo de la comunicación/educación. Trabajo Final de Grado. Facultad de Ciencias de la Comunicación. Universidad Nacional de Córdoba. Director. Dr. Enrique Bambozzi

10. Meirieu, Philippe. (2006). El significado de educar en un mundo sin referencias. Conferencia dictada el 22 de junio de 2006 en el Ministerio de Educación, Ciencia y Tecnología (Argentina). Mimeo.

11. Saviani, Dermeval (1982). Las teorías de la educación y el problema de la marginalidad en América Latina. Revista Argentina de Educación. Año II. № 3.

12. Silber, Julia. (1998). Pedagogía y Epistemología. Práctica educativa busca Pedagogía que la acompañe. En IV Encuentro de Cátedras de Pedagogía de Universidades Nacionales. Universidad Nacional de Río Cuarto.

13. Silber, Julia (2007). Recorridos recientes y trazos actuales de las tendencias pedagógicas en la Argentina. VI Encuentro Nacional de Cátedras de Pedagogía: Pedagogías desde América Latina: tensiones y debates contemporáneos. Universidad Nacional de la Patagonia Austral. Unidad Académica Caleta Olivia. Setiembre.

14. Schapira, Gabriel (2017). Subjetividades emergentes y producción textual me-diada por las TICS. Un estudio con adolescentes de 14 a 18 años en una escuela pública "urbano-periférica" del noreste de la ciudad de Córdoba, 2013-2014. MPEMT, CEA, UNC.

15. Valles Martínez, Miguel (2007). Técnicas cualitativas de investigación social. Reflexión metodológica y práctica profesional. Editorial Síntesis. Madrid.

16. Tiramonti, G (2011): "Escuela media: la identidad forzada" En Tiramonti (dir.) Variaciones sobre laforma escolar: límites y posibilidades de la escuela media. Buenos Aires: HomoSapiens.

17. Vanella, L y Maldonado, M. Cuadernos de Educación Año XII - No 12 - noviembre 2014 ISSN 2344-9152 1 CIFFyH - Universidad Nacional de Córdoba, Argentina La escolaridad secundaria: nuevas apuestas a la inclusión de los jóvenes. La experiencia del PIT 14-17 en la ciudad de Córdoba. 